Pflanzen. Flora, 80. 1896.

- Induzierte oder antonome Dorsiventralität bei Orchideenluftwurzeln? Biol. Centralbl. 35. 1915.

—_, Organographie der Pflanzen. Teil I, 1928, Teil III, 1933. Jena.

HAMmcnd, B. L., Regeneration of Podostemon ceratophyllum. Bot. Gaz. 97. 1936.

Imamura, S., Über die Dorsiventralität der unifazialen Blätter von Iris japonica Thunb. und ihre Beeinflussbarkeit durch die Schwerkraft. Memoir of Science, Kyoto Imp. Univ. Series B. 6. 1931.

JANCZEwskir, A., Organisation dorsiventrale dans les racines des Orchidées. Amn. des seiens nat. bot. VII Sér. 2. 1885 (zitiert nach Goebel 1915).

KonIBA, K., Observations on a Japanese species of Taeniophyllum. Proc. of the PanPacific Sience Congress, Tokyo. 2. 1926.

- Entwicklung, Wachstum und Organbildung der Pflanzen. 1931. Tokyo. (Japanisch).

Nabekichi, A., Über die Funktion der Luftwurzeln. Bot. Centralbl. 80. 1899.

Pfeffer, W., Studien über Symmetrie und spezifische Wachstumsursachen. Arb. d. bot. Inst. Würzburg. I 1871.

RAciborski, M., Biologisehe Mitteilungen aus Java. Flora, 85. 1898.

Vöchrting, H., Beiträge zur Morphologie und Anatomie der Ripsalideen. Jahrb. f. wiss. Bot. 9. 1873.

Über die Regeneration der Marchantien. Jahrb. f. wiss. Bot. 16. 1886.

\title{
Lokalisation der lichtempfindlichen Zonen von Keimorganen bei Avena sativa $\mathrm{L}$.
}

Von

\section{Toru Araki und Hideo Hamada.}

Mit 2 Textfiguren.

Eingegangen am 31. März 193\%.

\section{Einleitung.}

Bei der Untersuchung der Lichtwirkung auf die Pflanze hat man bisher meistenfalls eine ganze Pflanze oder ein Organ dem Licht ausgesetzt und daran die Lichtwirkung studiert. Als Hamada (1931) aber die Haferkoleoptile, deren Spitze ca. $2 \mathrm{~mm}$ aus dem Boden von Sägespänen herausgetreten war, mit kurzer Belichtung von oben her reizte, beobachtete er eine auffällige Wachstumshemmung des im Boden verdunkelten Mesokotyls und des Primärblattes. Diese hemmende Wirkung ist begreiflich basipetal geleitet worden. Es lässt sich nun die Frage aufstellen, wie das Wachstumsverhältnis verändert wird, wenn das darunter liegende 
Mesokotyl punktierend belichtet wird. Unsere vorliegenden Untersuchungen bezwecken nun durch die nachfolgenden Versuchsanordnungen die Lokalisation lichtempfindlicher Zonen in Koleoptile und Mesokotyl bei Hafer festzustellen.

Hier möchten wir unserem Lehrer, Herrn Prof. Dr. K. KoRIBA, der uns stets entgegenkommend geleitet und gestützt hat, unseren herzlichsten Dank aussprechen. Ebenfalls statten wir Herrn Dr. J. Maekawa von der Hokkaido Kaiserlichen Universität für seine freundliche Überlassung der Proben unseren verbindlichsten Dank ab.

\section{Methodik und Material.}

\section{(1) Versuchseinrichting.}

In zwei Dunkelschränken (Fig. 1) aus Blech $(25 \times 45 \times 30 \mathrm{~cm})$, die auf einer Messingbahn verschiebber sind, wurden drei 60 Watt Filamentlampen, welche ebenfalls vorn und hinten verschiebbar sind, parallel aufgestellt. Das Licht geht durch einen mit Alaunwasser gefüllten Filter von $10 \mathrm{~cm}$ Dicke und drei wagerecht gehaltene Röhrchen hindurch nach aussen. Am äusseren Ende jedes Röhrchens fügten wir je einen Deckel mit kleiner Öffnung ein (Fig. 1, D), von denen jeder je $0.5 \mathrm{~mm}$ Durchmesser besitzt und brachten diese dicht am Keimorgane an, damit wir das Organ punktierend gegenseitig, und zwar gleichhoch und geradlinig belichten könnten.

Die kleinen Blechkästen $(3.8 \times 3.8 \times 6.0 \mathrm{~cm})$ mit je einem Keimlinge befinden sich auf einem aufschraubbaren Gestell, um eine beliebige Stelle des Keimlings dem Licht auszusetzen.

\section{(2) Material.}

Als Material wurden die Hafersamen „Clydesdale“" aus der Hokkaido Universität herangezogen. Jeder entspelzte Same wurde in dem eben erwähnten kleinen Kästchen mit Sacursscher Nährlösung einzeln ausgesät, bei $30^{\circ} \mathrm{im}$ Dunkeln gekeimt und weiter grossgezogen. Sechs oder zwölf von diesen Kästchen als ein Satz wurden in einen grossen Kasten $(15 \times 18.5 \times 8 \mathrm{~cm})$ mit Deckel hineingelegt und im Schrank aufgestellt und bei jedem Versuche wurden die Keimlinge von

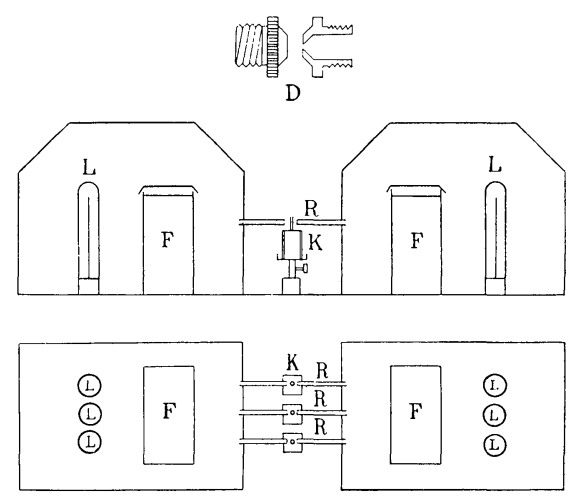

Fig. 1. Der Punktierbelichter. $\times 1 / 20$. Oben: Seitenansicht, unten: Oberansicht. L. Filamentlampe, F. Filter, R. Röhrchen, K. Kästchen, D. Deckel mit kleiner Öffnung (Seitenansicht und Längsschnitt $\times 1 / 2)$. 
ein und demselben Kasten verwendet. Alle Behandlungen erfolgten unter einer 1 Watt Rotlampe.

\section{(3) Belichtung der Keimorgane.}

Jeder Keimling, dessen Mesokotyl resp. Koleoptile erst $6 \mathrm{~mm}$ Länge erreicht hatten, wurde je nach dem Versuche, bald der Ober-, bald der Mittel- und bald der Unterteil, mit $50 \mathrm{MK} \times 15$ Minuten belichtet ${ }^{11}$. Es gibt also betreffs der Belichtungsteile 6 Versuchsreihen. Am Ende des Versuches wurden die Keimlinge in Alkohol fixiert. Nach der Messung der dreierlei Organe wurde die Hemmung, bzw. die Beschleunigung der gereizten Keimlinge im Vergleich mit denjenigen der zugleich angestellten Kontrollpflanzen berechnet.

\section{Resultate.}

\section{(1) Kontrollnersuche.}

Um den Wachstumsverlauf der Keimorgane zunächst im Dunkeln zu verfolgen, wurde eine Reihe Keimlinge jeden Tag nacheinander herausgenommen und in Alkohol fixiert. Der tägliche Verlauf ergibt wie üblich eine S-Kurve und zwar streckt sich zuerst das Mesokotyl, dann die Koleoptile mit eintägiger Verschiebung mit gleichartiger Kurve und zuletzt das Primärblatt im Alter von 4 Keimtagen mit einer weitaus steilen S-Kurve.

Um die Versuchsbedingungen möglichst gleich zu halten, wurde die Kontrolle gleichzeitig mit den belichteten Pflanzen aus dem grossen Kasten herausgenommen, in freie Luft in den Keller verlegt und nach der Belichtung der Versuchspflanze wurden sie gleich wieder in den grossen Kasten zurückgebracht. Die Endlänge dieser Kontrollpflanzen im 120stündigen Alter ist wie folgt:

Tabelle 1. Endlänge der Kontrollpflanzen.

\begin{tabular}{|c|c|c|c|}
\hline Mesokotyl. & Koleoptile. & Rrimärblatt. & Zahl d. Keimlinge. \\
\hline $46.3 \mathrm{~mm}$ & $48.4 \mathrm{~mm}$ & $81.9 \mathrm{~mm}$ & 314 \\
\hline
\end{tabular}

Da die Hemmungsgrösse des Wachstums von Mesokotyl durch Licht im Alter von 5 Tagen als Endresultate am stärksten auftritt (HAmadA 1931, l.c.), wurde auch die Wachstumsgrösse von übrigen Organen in demselben Alter gemessen.

1) Nach Brunner (1936) soll der Absorptionsgrad dés Lichtes durch Koleoptile und Mesokotyl verschieden sein, aber es ist noch nicht entschieden, welcher Prozentsatz auf das Wachstum von Wirkung ist. 


\section{(2) Belichtungsversuche.}

Die lichtempfindlichste Periode von Avena-Mesokotyl befindet sich im Alter von 36 Stunden. Das Mesokotyl erreicht aber zu der Zeit 1-2 mm Länge, so dass dabei keine Punktierbelichtung ausführbar ist. Daher mussten wir warten, bis die Keimorgane sich ca. 5-6 mm strecken, nämlich bis zum 54stündigen Alter. In diesem Stadium sind jedoch die Keimlinge noch genügend empfindlich. Das Mesokotyl und die Koleoptile erleiden durch das Licht fast ausschliesslich Wachstumshemmung, während das Primärblatt umgekehrt eine Förderung erfährt.

(a) Belichtung der Koleoptilenteile.

Durch 15 Minuten lange Punktierbelichtung auf je drei Koleoptilenteilen kamen wir zu den folgenden Resultaten:

Tabelle 2. Hemmungs- (-) resp. Förderungs-Prozente $(+)$ bei der Koleoptilenbelichtung.

\begin{tabular}{|c|c|c|c|c|c|c|c|c|c|}
\hline \multirow{3}{*}{$\begin{array}{c}\text { Bclichtungs- } \\
\text { teil. }\end{array}$} & \multicolumn{9}{|c|}{ Organ. } \\
\hline & \multicolumn{3}{|c|}{ Mesokotyl. } & \multicolumn{3}{|c|}{ Koleoptile. } & \multicolumn{3}{|c|}{ Primärblatt. } \\
\hline & $\begin{array}{l}\text { Vers. } \\
\text { A }\end{array}$ & $\begin{array}{l}\text { Vers. } \\
\mathrm{B}\end{array}$ & $\begin{array}{l}\text { Durch- } \\
\text { schnitt }\end{array}$ & $\begin{array}{l}\text { Vers. } \\
\text { A }\end{array}$ & $\begin{array}{l}\text { Vers. } \\
\mathrm{B}\end{array}$ & $\begin{array}{l}\text { Durch- } \\
\text { schnitt }\end{array}$ & $\begin{array}{l}\text { Vers. } \\
\text { A }\end{array}$ & $\begin{array}{c}\text { Vers. } \\
\text { B }\end{array}$ & $\begin{array}{l}\text { Durch- } \\
\text { schnitt }\end{array}$ \\
\hline Obes: & $-12.6 \%$ & $-14.6 \%$ & $-13.6 \%$ & $-4.0 \%$ & $-2.3 \%$ & $-3.2 \%$ & $-1.8 \%$ & $-25.3 \%$ & $-13.6 \%$ \\
\hline Mittel & -19.7, & -19.4, & -19.6, & -5.2, & -6.7, & -6.2, & +25.8, & +64.4, & +45.1, \\
\hline Unter & -28.4, & -27.6, & -28.0, & 0 , & +2.7 & +1.4, & +34.3, & +7.9, & +21.1, \\
\hline
\end{tabular}

Wie man sieht, erleidet das Mesokotyl durch Belichtung der Koleoptile seine bedeutendste Hemmung, und zwar weist die im Unterteil gegebene Belichtung die stärkste Sistierung auf, während auf das Wachstum der Koleoptile selbst die Wirkung nicht namhaft ist. Das Primärblatt erleidet bei der Oberteilbelichtung eine schwache Hemmung, während es bei Mittelund Unterteilbelichtung eine üppige Beschleunigung erfährt.

\section{(b) Belichtung der Mesokotylteile.}

Wie die nachstehenden Resultate zeigen, ist bei der 15 Minuten langen Mesokotylbelichtung die Hemmung des Mesokotyls selbst sehr auffallend, ganz besonders bei der Obertelbelichtung, während bei der Unterteilbelichtung jedoch die Hemmung nur ungefähr 10\% der letzteren beträgt (Tab. 3).

Für die Streckung der Koleoptile zeigen aber die drei verschiedenen Mesokotylbelichtungen keine eindeutige Wirkung; sie erfährt bald eine Hemmung, bald eine Förderung. Dies beruht vielleicht darauf, dass die korrelative Wachstumswirkung oft stärker ausfällt als die unmittelbare 
Tabelle 3. Hemmungs-(-) resp. Förderungs-Prozente $(+)$ bei der Mesokotylbelichtung.

\begin{tabular}{|c|c|c|c|c|c|c|c|c|c|}
\hline \multirow{3}{*}{$\begin{array}{l}\text { Belichtungs- } \\
\text { teil. }\end{array}$} & \multicolumn{9}{|c|}{ Organ. } \\
\hline & \multicolumn{3}{|c|}{ Mesokotyl. } & \multicolumn{3}{|c|}{ Koleoptile. } & \multicolumn{3}{|c|}{ Primärblatt. } \\
\hline & $\begin{array}{l}\text { Vers. } \\
\text { A }\end{array}$ & $\begin{array}{l}\text { Vers. } \\
\mathrm{B}\end{array}$ & $\begin{array}{l}\text { Dureh- } \\
\text { schnitt }\end{array}$ & $\begin{array}{l}\text { Vers. } \\
\text { A }\end{array}$ & $\begin{array}{l}\text { Vers. } \\
\text { B }\end{array}$ & $\begin{array}{l}\text { Durch- } \\
\text { schnitt }\end{array}$ & $\begin{array}{c}\text { Vers. } \\
\mathrm{A}\end{array}$ & $\begin{array}{l}\text { Vers. } \\
\mathrm{B}\end{array}$ & $\begin{array}{l}\text { Durch- } \\
\text { schnitt }\end{array}$ \\
\hline Ober & $-32.2 \%$ & $-21.0 \%$ & $-26.6 \%$ & $-2.7 \%$ & $-1.8 \%$ & $-2.3 \%$ & $+17.0 \%$ & $+41.7 \%$ & $+29.3 \%$ \\
\hline Mittel & -11.6, & -12.2, & -11.9, & +12.5, & $+1 . \bar{y}$, & +7.0 & $+24 . ?$, & +31.8, & +28.0, \\
\hline Unter & -2.4, & -2.8, & -2.6, & -1.6, & -1.9, & -1.8, & +8.2, & +57.8, & +32.9 , \\
\hline
\end{tabular}

Lichtwirkung. Bei dem Primärblatt ergibt sich gewöhnlich blosse Beschleunigung, deren Grad desto grösser ausschlägt, je mehr die Belichtung sich dem oberen Teil des Mesokotyls zuwendet. Die Wachstumsförderung des Primärblattes steht also in korrelativ umgekehrtem Verhältnis wie die Hemmung des Mesokotyls.

\section{Diskussion.}

\section{(1) Lokalisation des Hemmungseffektes.}

Die maximale Hemmungswirkung für das Mesokotyl liegt in seinem Oberteil und in dem Unterteil der Koleoptile, nämlich in der Nähe des Knotens der Mesokotylobergrenze. Die Belichtung der Mittel- und Oberteile der Koleoptile zeigt nur eine mässige Hemmung auf das Mesokotyl und die minimale Sistierung des Mesokotyls ist durch Belichtung von Mittel- und Unterteilen des Mesokotyls selbst zu sehen.

Die sich am lebhaftesten streckende Zone des Mesokotyls liegt am obersten Teil, wo man in ziemlich späteren Entwicklungstadien noch die Zellteilungsfiguren beobachten kann, während in den Mittel- und Unterteilen die Zellteilung bald aufhört und nur die Zellstreckung vor sich geht (Avery und Burkholder 1936, Araki, unveröffentlicht). Es ist daher ohne Zweifel, dass die Belichtung des Oberteils des Mesokotyls oder des Unterteils der Koleoptile, der sich unmittelbar dem Mesokotyl anschliesst, das Mesokotylwachstum am stärksten beeinflusst, indem das Licht im ersten Falle unmittelbar und im letzteren mittelbar durch das halbdurchsichtige Koleoptilengewebe hindurch einwirkt. Die Wachstumshemmung der Koleoptile ist jedenfalls nicht sehr auffallen und selten kann man sogar infolge der korrelativen Wirkung eine recht schwache Förderung sehen. Eigentlich hört die Zellteilung der Koleoptile schon bald nach dem Heraustreten aus der Erde auf, aber der Ort maximaler Streckung bei $15 \mathrm{~mm}$ langen Keleoptilen findet sich ca. $12 \mathrm{~mm}$ von der Spitze. Später geht er aber in den oberen Teil der Koleoptile über (Avery und Burkholder 
1936, Jost u. Reiss, 1937). Aus diesem Grunde ist es selbstverständlich, dass die Koleoptile bei der Belichtung in noch jüngerem Alter wie bei unserem Versuchsmaterial ihre maximale Wachstumszone zwischen dem Mittel- bzw. dem Oberteil haben muss. Da aber bei der Koleoptile die Wuchsstoffe in ihrer Spitze erzeugt werden, sollte die Sache hier von noch komplizierterer Natur sein (LnNae 1927). Das Wachstum von Primärblatt wird zuletzt durch die Belichtung im allgemeinen fördernd beeinflusst, ausgenommen, wenn die Koleoptilenspitze belichtet wird.

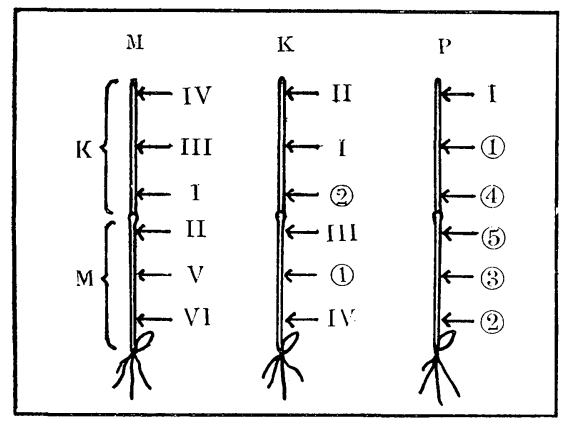

Fig. 2. Schematische Darstellung der Lokalisation lichtempfindlicher Zonen bei den Keimorganen. Die Endresultate von Mesokotyl(M), Koleoptile(K) und Primärblatt( $\left({ }^{\prime}\right)$ durch die $15 \mathrm{Min}$. lange Belichtung. I-VI: Ordnung der Hemmungsgrösse, (1) (5): Ordnung der Förderungsgrösse, $\leftarrow$ : Belichtungsort der Keimorgane.

(2) Wachstumskorrelation bei der Belicit'tung.

Nach den Untersuchungen von BEYER (1928), WENT (1928), vaN DER Weis (1932) mit Avenu-Koleoptile und von van Overbeen (1933) mit Raphanus-Hypokotyl ist es wohl bekannt, dass der 'Transport der pflanzlichen Wuchsstoffe in der basipetalen Richtung vor sich geht ${ }^{1)}$, unabhängig von seinem Mechanismus, sei es Diffusion, Kataphorese, Protoplasmaströmung oder aktiver plasmatischer Transport und dass die Leitung durch das Licht beeinflusst wird und dadurch auch das Wachstum.

Nach unseren Ergebnissen ist der Einfluss des Lichtes auf Mesokotylwachstum bei der Belichtung des Mesokotylunterteils aufiallend schwach, der Lichtreiz kann demnach nicht akropetal transportiert worden sein und nur auf einen schmalen Teil des Mesokotyls wachstumshemmend eingewirkt haben.

In Gegensatz dazu wirkt die Belichtung auf die Mesokotyloberteile nicht nur unmittelbar auf die belichtete schnellst wachsende Zone, sondern auch auf die übrigen Teile indirekt hemmend ein, sodass dadurch das Mesokotyl auffällig in kurzem Zustande bleibt.

Ähnlicherweise wird der Lichtreiz bei der lokalen Belichtung der Koleoptile nach dem Mesolkotyl basipetal geleitet und dadurch die

1) Snow (1936) fand, dass der akropetale Transport von Wuchsstoffen auch vorkommt, wemn die Paste angestellt wird. (Cf. Jos't 1937, Jost u. Reiss 1937) 
Streckung des Mesokotyls ziemlich stark gehemmt. Dabei ist der Wirkungsgrad desto grösser, je näher der Belichtungsort der Streckungszone (d.h. dem Mittelteil) liegt.

Es ist aber noch nicht entschieden, dass bei lokaler Belichtung des Mesokotyls die Koleoptile nur schwache Hemmung und das Primärblatt meistens eine Beschleunigung erfahren muss. Im letzteren Falle könnte man stofflich so erklären, dass die Baustoffe, die sonst beim Wachstum des Mesokotyls verbraucht werden sollten, hier für die Entwicklung des Primärblattes geliefert sind (cf. LaIbach 1936, CoRdes u. LAIBACH 1936).

Zum Schluss sei noch darauf hingewiesen, dass die Belichtung des oberen Mesokotylteils einen auffallenden Hemmungseinfluss auf das Organ selbst und zwar nicht nur die Streckung, sondern auch auf die Zellteilung ausübt. Es ist also sehr wahrscheinlich, dass im genannten Teile ein Zellteilungshormon vorhanden ist, das aber anders als bei denjenigen der Koleoptilenspitze durch das Licht nicht nur direkt, sondern auch wie bei der Koleoptilenbelichtung indirekt zerstört, zu werden scheint.

Botanisches Institut der Kaiserl. Universität zu Kyoto.

\section{Literaturverzeichnis.}

Averi, G. S. and Burkholder, P. R., (1936). Polarized growth and cell studies on the Avena coleoptile, phytohormone test object. Bull. Torrey Bot. Club. 63. 1.

Beyer, A., (1928). Beiträge zum Problem der Reizleitung. Zeitschr. f. Bot. 20.

Brunner, T., (1936). Über die optischen Eigensehaften phototroper Keimlinge. Planta, 26. 1.

Cordes, H. und F. Laibach, (1936). Über die Abhängigkeit des Wachstums von der Assimilation. Jahrb. f. wiss. Bot. 84. 1/2.

Hamada, H., (1931). Über die Beeinflussung des Wachstums des Mesokotyls und der Koleoptile von Avena-Keimlingen dureh das Licht. Mem. Coll. Sci. Kyoto. Series B. 6. 4.

Jost, L., (1937). Über Wuehsstoffe. Zeitschr. f. Bot. 31. 2.

Jost, L. und Elisabeth Rfiss, (1937). Zur Physiologie der Wuchsstoffe III. Zeitschr. f. Bot. 31. 2.

IAABBACH, F., (1936). Über den Einfluss auf das Reaktionsvermögen der Pflanze gegenüber Wuchsstoff. Jahrb. f. wiss Bot. 83. 2.

Lange, S., (1927). Die Verteilung der Lichtempfindlichkeit in der Spitze der Haferkoleoptile. Jahrb. f. wiss. Bot. 65. 1.

Overberk, J. VAN, (1933). Wuchsstoff, Lichtwachstumsreaktion und Phototropismus bei Raphanus. Ree. trav. bot. néerl. 30. 537.

Snow, R., (1936). Upward effects of auxin in coleoptiles and stems. New Phyt. 35. 4.

WEIJ, H. G. VAN DER, (1932). Der Mechanismus des Wuchsstofftransportes. Rec. trav. bot. néerl. 29. 379.

Went, F. W., (1928). Wuchsstoff und Wachstum. Ree. trav. bot. néerl. 25. 1. 